\title{
Decision Making Around Optimal Management of Liver-Limited Metastatic Colorectal Cancer
}

\author{
Robert P. Jones, BSc, MBChB, PhD ${ }^{1,2}$ and Graeme J. Poston, MBChB, DSc, FRCS ${ }^{2}$ \\ ${ }^{1}$ School of Cancer Studies, Institute of Translational Medicine, University of Liverpool, Liverpool, UK; ${ }^{2}$ Liverpool \\ Hepatobiliary Unit, Aintree University Hospital, Liverpool, UK
}

Since the early 1980s, selected patients with liver-limited colorectal metastases (CRLM) have increasingly been offered hepatic resection with the aim of improving longterm survival. Meta-analysis suggests approximately $40 \%$ of patients undergoing surgery are alive 5 years later, with $25 \%$ surviving for 10 years. ${ }^{1}$ These impressive results have established hepatic resection as a key treatment modality for CRLM. Patients are now considered technically resectable if all disease can be removed with microscopically negative margins whilst maintaining a sufficient volume of liver parenchyma. However, it is also clear that not all patients with technically resectable liver metastases enjoy long-term survival after surgery; within 1 year approximately $30 \%$ will develop recurrence and $15 \%$ will succumb to their disease. ${ }^{2}$

While there is broad agreement among liver surgeons over which patients clearly should and should not be offered resection, we have demonstrated that in the UK there is a lack of consensus between liver surgeons and oncologists. ${ }^{3}$ For a patient with a small solitary liver metastasis presenting several years after primary resection, surgery is generally accepted to offer a clear and significant long-term survival benefit. For patients with synchronous extensive large-volume disseminated disease, surgery is generally considered inappropriate. Deciding on the optimal management strategy for patients who fall into the gray area between these two groups is one of the most challenging decisions in the management of CRLM and

(C) Society of Surgical Oncology 2015

First Received: 30 October 2015;

Published Online: 2 December 2015

R. P. Jones, BSc, MBChB, PhD

e-mail: robjones@liv.ac.uk involves careful assessment of both technical and oncological factors.

In this timely analysis, Choti et al. ${ }^{4}$ identified clear consensus between specialist hepatic surgeons about the technical resectability of patients with liver metastases. Exploring medical oncologists' perceptions of resectability, cases considered easily resectable by specialist surgeons were considered resectable by only $34 \%$ of oncologists. This highlights the critical role of surgeons in defining what constitutes technically resectable disease. A further $42 \%$ of oncologists suggested these patients were potentially convertible to resection, suggesting an enthusiasm for systemic chemotherapy prior to surgery. This may of course reflect inherent bias-surgeons prefer to offer surgery, oncologists prefer to offer chemotherapy. It may also be attributable to oncologists perceiving a higher oncological risk for these patients, where a trial of systemic therapy to attain disease control may offer a better assessment of tumor biology and gauge likely long-term benefit after surgery. The role of neoadjuvant chemotherapy for easily resectable CRLM remains contentious, ${ }^{5,6}$ and therefore deciding which patients should be offered initial systemic therapy and which should proceed straight to surgery remains far from clear. Hence, one important finding of the study is that oncologists and surgeons differ in their perception of where the gray area between clearly resectable and irresectable disease lies, highlighting the critical importance of joint decision making.

Another striking finding from the study was the low levels of referral for consideration of surgery, despite $78 \%$ of oncologists having easy access to multidisciplinary case conferences and almost $40 \%$ regularly participating in these conferences, irrespective of whether they themselves had a patient for discussion. Request for a surgical consultation was associated with an extremely low likelihood of oncologists classifying a case as initially unresectable, a 
critical point considering the already highlighted difference between what oncologists perceive to be technically resectable and what is considered resectable by specialist hepatobiliary surgeons. Perhaps more importantly, even when oncologists considered patients initially resectable, only $88 \%$ would refer for surgery. These low referral rates suggest a significant number of patients with easily resectable disease, in whom surgery would generally be accepted as best management, are being denied potentially curative treatment.

Multidisciplinary discussion and decision making is well-recognized as the optimal approach for the management of patients with CRLM, ${ }^{7}$ and the study by Choti et al. $^{4}$ clearly highlights the importance of surgeon and oncologist working together to achieve the best outcome for these complex patients. In the UK, wide variations in referral patterns for consideration of liver resection have been recognized, with a tenfold variation reported between the highest and lowest referring centers. ${ }^{8}$ This variation has led the UK National Institute for Health and Care Excellence (NICE) to publish national guidance stipulating that all patients with liver-limited metastatic colorectal cancer should be discussed at a multidisciplinary case conference involving oncologists and specialist liver surgeons. ${ }^{9}$ The importance of such multidisciplinary decision making has been further reinforced by growing evidence suggesting benefit from alternative nonresectional interventions for irresectable CRLM, as highlighted by the recently updated results from EORTC 40004, which demonstrated a significant improvement in overall survival for patients treated with ablation of liver metastases and systemic chemotherapy versus chemotherapy alone. ${ }^{10}$

Further work is needed to better identify patients likely to benefit most from liver resection. Existing prognostic markers rely on gross assessment of pathology as a surrogate for underlying tumor biology. It seems likely that increased understanding of tumor biology will improve our stratification of patients, improving outcomes after hepatectomy by allowing better decision making about which patients will enjoy long-term benefit. As Choti et al. ${ }^{4}$ have clearly demonstrated, it is crucial that any such decision making should take place in a multidisciplinary environment.

\section{REFERENCES}

1. Kanas GP, Taylor A, Primrose JN, et al. Survival after liver resection in metastatic colorectal cancer: review and meta-analysis of prognostic factors. Clin Epidemiol. 2012;4:283-301.

2. Jones RP, Jackson R, Dunne DF, et al. Systematic review and meta-analysis of follow-up after hepatectomy for colorectal liver metastases. Br J Surg. 2012;99(4):477-86.

3. Jones RP, Vauthey JN, Adam R, et al. Effect of specialist decision-making on treatment strategies for colorectal liver metastases. Br J Surg. 2012;99(9):1263-9.

4. Choti MA, Thomas M, Wong SL, et al. Surgical resection preferences and perceptions among medical oncologists treating liver metastases from colorectal cancer. Ann Surg Oncol. 2015. doi:10. 1245/s10434-015-4925-1.

5. Nordlinger B, Sorbye H, Glimelius B, et al. Perioperative chemotherapy with FOLFOX4 and surgery versus surgery alone for resectable liver metastases from colorectal cancer (EORTC Intergroup Trial 40983): a randomised controlled trial. Lancet. 2008;371(9617):1007-16.

6. Primrose J, Falk S, Finch-Jones M, et al. Systemic chemotherapy with or without cetuximab in patients with resectable colorectal liver metastasis: the new EPOC randomised controlled trial. Lancet Oncol. 2014;15(6):601-11.

7. Adam R, De Gramont A, Figueras J, et al. The oncosurgery approach to managing liver metastases from colorectal cancer: a multidisciplinary international consensus. Oncol. 2012;17(10): 1225-39.

8. Morris EJA, Forman D, Thomas JD, et al. Surgical management and outcomes of colorectal cancer liver metastases. Br J Surg. 2010;97(7):1110-1118.

9. Poston J, Tait D, O'Connell S, Bennett A, Berendse S, On behalf of the Guideline Development Group. Diagnosis and management of colorectal cancer: summary of NICE guidance. BMJ. 2011;343:d6751.

10. Ruers T, Punt CJA, van Coevorden F, et al. Radiofrequency ablation (RFA) combined with chemotherapy for unresectable colorectal liver metastases (CRC LM): long-term survival results of a randomised phase II study of the EORTCNCRI CCSG-ALM Intergroup 40004 (CLOCC). Ann Oncol. 2015;26 Suppl 4:iv114-5. 